\title{
PSEUDOGROUPS OF TRANSFORMATIONS ON DISCRETE TOPOLOGICAL SPACES
}

\author{
Jolanta Lipińska \\ Institute of Mathematics, Czestochowa University of Technology \\ Częstochowa, Poland \\ jolanta.lipinska@im.pcz.pl
}

\begin{abstract}
In all the earlier papers pseudogroups of transformations, generalised inverse semigroups and their connections were considered. This paper is a kind of recapitulation of these problems. Considering pseudogroups of transformations on discrete topological spaces is a step in the same direction. In this paper we notice that domains of functions belonging to a pseudogroup of transformations on discrete topological spaces - when we join an empty set to them create not only a topological space but also a $\sigma$-body. We also consider pseudogroups on discrete topological spaces with the finite number of elements. The third problem is the influence of topology on relation of partial order.
\end{abstract}

Keywords: pseudogroups of transformations, generalised inverse semigroups

\section{Introduction}

The notion of a pseudogroup was formed progressively together with the development of differential geometry. The first mathematicians who realized that a classic notion of a group of transformations was not sufficient for purposes of differential geometry were O. Veblen and J.H.C. Whitehead in 1932. Their definition was improved by J.A. Schouten and J. Haantjes in 1937, S. Gołąb in 1939 and C. Ehresmann in 1947. Both Gołąb's and Ehresmann's definitions are good enough to be used at present. It was shown in [1] that axioms of Ehresmann's definition can be formulated in an equivalent way which simplify proofs. We used this definition in [2] to show that a group of transformation can be treated as a pseudogroup. Inverse semigroups and other generalizations of the notion of a group are present not only in differential geometry [3,4]. This theory is still being developed [5].

\section{Main results}

Let us recall the following version of Ehresmann's definition which is used in differential geometry and can be found in [6]. 
Definition 1. A pseudogroup of transformations on a topological space $S$ is a set $\Gamma$ of transformations satisfying the following axioms:

$1^{\circ}$ Each $f \in \Gamma$ is a homeomorphism of an open set of $S$ onto another open set of $S$;

$2^{\circ}$ If $f \in \Gamma$, then the restriction of $f$ to an arbitrary open subset of the domain of $f$ is in $\Gamma$;

$3^{\circ}$ Let $U=\bigcup_{i} U_{i}$ where each $U_{i}$ is an open set of $S$. A homeomorphism $f$ of $U$ onto an open set of $S$ belongs to $\Gamma$ if the restriction of $f$ to $U_{i}$ is in $\Gamma$ for every $i$;

$4^{\circ}$ For every open set $U$ of $S$, the identity transformation of $U$ is in $\Gamma$;

$5^{\circ}$ If $f \in \Gamma$, then $f^{-1} \in \Gamma$;

$6^{\circ}$ If $f \in \Gamma$ is a homeomorphism of $U$ onto $V$ and $g \in \Gamma$ is a homeomorphism of $Y$ onto $Z$ and if $V \cap Y$ is non-empty, then the homeomorphism $g \circ f$ of $f^{-1}(V \cap Y)$ onto $g(V \cap Y)$ is in $\Gamma$.

We will also use the following definition introduced in [1].

Definition 2. A non-empty set $\Gamma$ of functions, for which domains $D_{f}$ are arbitrary non-empty sets, will be called a pseudogroup of functions if it satisfies the following conditions:

$1^{\circ} f\left(D_{f}\right) \cap D_{g} \neq \varnothing \Rightarrow g \circ f \in \Gamma$ for $f, g \in \Gamma$,

$2^{\circ} f^{-1} \in \Gamma$ for $f \in \Gamma$,

$3^{\circ} \bigcup \Gamma^{\prime} \in \Gamma$ for $\Gamma^{\prime} \in\langle\Gamma\rangle$

where

$$
\langle\Gamma\rangle=\left\{\varnothing \neq \Gamma^{\prime} \subset \Gamma: \cup \Gamma^{\prime} \text { is a function and } \bigcup\left(\Gamma^{\prime}\right)^{-1} \text { is a function }\right\}
$$

and

$$
\left(\Gamma^{\prime}\right)^{-1}=\left\{f^{-1}: f \in \Gamma^{\prime}\right\}
$$

and $f^{-1}$ denotes an inverse relation.

It was shown in [1] that if $\Gamma$ is a pseudogroup, then $\left(\bigcup_{f \in \Gamma} D_{f},\left\{D_{f}: f \in \Gamma\right\} \cup\{\varnothing\}\right)$ is a topological space and $\Gamma$ is an Ehresmann pseudogroup of transformations on this topological space. On the other hand, if $\Gamma$ is an Ehresmann pseudogroup of transformations on a topological space $S$, then $\Gamma$ is a pseudo-group of functions. 
We will use the following definition which we can find in [7]

Definition 3. We will say that $\Gamma$ is a Schouten-Haantjes pseudogroup if it satisfies the following axioms:

$1^{\circ}$ If $f \in \Gamma, g \in \Gamma$ and $g \circ f$ is defined, then $g \circ f \in \Gamma$,

$2^{\circ}$ If $f \in \Gamma$ and $f^{-1}$ is defined, then $f^{-1} \in \Gamma$.

We notice that a Schouten-Haantjes pseudogroup does not satisfy axioms of Definition 1 because the union of domains may not be a domain of a function belonging to it. In this case we can only say that an intersection of a finite numbers of domains will be a domain of a function which belongs to a Schouten-Haantjes pseudogroup.

Definition 4. A generalized inverse semigroup is a partial groupoid $(B, \bullet)$ satisfying the following axioms:

$1^{\circ} a \bullet(b \bullet c)=(a \bullet b) \bullet c$

holds when one of the sides is defined;

$2^{\circ}$ For every $a \in B$ there exists exactly one $b \in B$ such that

$$
a \bullet(b \bullet a)=a \text { and } b \bullet(a \bullet b)=b
$$

We will also need the following definitions and denotations for elements of a generalised inverse semigroup $(B, \bullet)$ which were introduced in [8]. We will write $a b$ instead of $a \bullet b$. For every $a \in B$ the only one $b \in B$ from $2^{\circ}$ of Definition 4 will be denoted by $a^{\prime}$ and called a generalised inverse element of $a, a^{\prime} a$ will be called a right identity of $a$ and $a a^{\prime}$ a left identity of $a$. It is obvious that $a$ will be then a generalised inverse element of $a^{\prime}, a^{\prime} a$ will be a left identity of $a^{\prime}$ and $a a^{\prime}$ a right identity of $a^{\prime}$. If $a$ is a right and left identity for all elements of $B$ we say that $a$ is an identity. We will say that $a \in B$ is an idempotent element when $a a=a$. It was shown in [8] that $a=a^{\prime}$ for an idempotent element $a$, so it means that its generalised inverse element, the right and left identity are all equal to $a$. It was also shown that the following relation

$$
a \leq b \Leftrightarrow b a^{\prime} a=a
$$

is a partial order in a generalised inverse semigroup. To prove it we used a lemma saying that if $a, b$ are idempotent elements, the operation $a b$ is commutative.

It was proved in [9] that we can obtain an inverse semigroup from every generalised inverse semigroup $(B, \bullet)$ joining an element $O \notin B$. Then $(B \cup\{O\}, *)$ is a semigroup where the operation $*$ is defined in the following way:

$a * b=a b$ when the operation $a b$ is defined,

$O$ in the other case. 
We will also use the theorem which was proved in [10] and says that if $\Gamma$ is a pseudogroup of transformations on a topological space $S$, then $\Gamma$ is a generalised inverse semigroup with identity. Of course we can replace a pseudogroup of transformations by a pseudogroup of functions and the theorem will be true. It was shown in [10] that even a Schouten-Haantjes pseudogroup is a generalised inverse semigroup. As the definition of Schouten-Haantjes is more general, we can also say that a pseudogroup of functions is a generalised inverse semigroup. It was also proved in [11] that every generalised inverse semigroup is isomorphic to a Schouten-Haantjes pseudogroup.

Now we will formulate the problems. What can we say when we consider pseudogroups on discrete topological spaces? What can we say when we consider pseudogroups on discrete topological space with a finite number of elements? Is there any influence of topology on algebraic structure as it was in the case of antidiscrete topology [12]. We can formulate the following theorems.

Theorem 1. Domains of functions belonging to an Ehresmann pseudogroup on a discrete topological space together with the empty set create a $\sigma$-body.

Proof. As it was shown in [1], the domains with the empty set consist a topology for all pseudogroups of functions but it is not enough. It will be a $\sigma$-body because in a case discrete topological space this family consists of all subsets of the given set which is of course enough to be a $\sigma$-body.

Theorem 2. We can obtain an Ehresmann pseudogroup on a discrete topological space with the finite number of elements joining to every cycling group the restrictions its elements to all subsets.

Proof. We have to check all axioms of the definition of a pseudogroup which is not difficult. Of course it is better to use Definition 2. We get what we need using axioms of a group.

Theorem 3. If a generalised inverse semigroup is isomorphic with a pseudogroup of transformations on a discrete topological space then for any idempotent exists smaller or equal to it idempotent for which does not exist smaller idempotent.

Proof. These idempotents for which a smaller idempotent does not exist will be of course idempotents isomorphic to transformations on singletons.

\section{Conclusions}

It was shown in [13] that for generalized inverse semigroups isomorphic to a pseudogroup of transformations on a topological space there exists the largest element in the set of idempotent elements. For pseudogroups of transformations 
on discrete topological spaces, it is also possible to notice the influence of topology on relation of partial order. Of course Theorem 3 is not true for all pseudogroups. For example, it is not true for pseudogroup of transformations defined on the space of real numbers with natural topology.

\section{References}

[1] Lipińska J., Diffeomorphisms of quasi-algebraic spaces, Demonstratio Mat. 1986, 19, 139-151.

[2] Lipińska J., Groups of transformations as pseudogroups of functions, Prace Naukowe Instytutu Matematyki i Informatyki 2006, 1(5).

[3] Kubarski J., Exponential mapping for Lie groupoids, Colloquium Mathematicum 1982, XLVII, Fasc. 2, 268-282.

[4] Odzijewicz A., Sliżewska A., Groupoids and inverse semigroups associated to $\mathrm{W}^{*}$-algebras, Cornell University Library 2011, 1-43, http://arxiv.org/pdf/1110.6305.pdf

[5] Srinivas K.V.R., Nandakumar R., Algebraic properties and examples of inverse semigroups, Proyecciones Journal of Mathematics 2009, 28, 3, 227-232.

[6] Kobayashi S., Nomizu K., Foundations of Differential Geometry I, New York, London 1963.

[7] Kucharzewski M., Elementy teorii obiektów geometrycznych, U. Śl., Katowice 1969.

[8] Lipińska J., Uogólniona półgrupa inwersyjna i jej własności, Zeszyty Naukowe Pol. Śl. Mat.-Fiz. 1981, 39, 287-295.

[9] Lipińska J., Stosunek uogólnionej półgrupy inwersyjnej do innych systemów algebraicznych z jedną dwuargumentową częściową operacją, Zeszyty Naukowe Pol. Śl. Mat.-Fiz. 1981, 39, 305-310

[10] Lipińska J., Przykłady uogólnionych półgrup inwersyjnych, Zeszyty Naukowe Pol. Śl. Mat.-Fiz. 1981, 39, 297-303.

[11] Lipińska J., Algebraizacja pseudogrupy Schoutena-Haantjesa, Zeszyty Naukowe Pol. Śl. Mat.-Fiz. 1981, 39, 311-317.

[12] Lipińska J., Pseudogroups of transformations on antidiscrete topological spaces, Scientific Research of the Institute of Mathematics and Computer Science 2012, 3(11), 93-97.

[13] Lipińska J., Properties of partial order in generalised inverse semigroups, Scientific Research of the Institute of Mathematics and Computer Science 2011, 1(10), 145-149. 